\title{
Valoración del uso de la plataforma virtual Moodle como recurso pedagógico en la enseñanza universitaria de la informática
}

\author{
Juan Manuel Claro-Vásquez ${ }^{\text {a* }}$ \\ aMagíster en prácticas pedagógicas, Universidad de Santander (UDES), Cúcuta \\ Forma de citar: Claro, J. M. (2017). Valoración del uso de la plataforma virtual Moodle como recurso \\ pedagógico en la enseñanza universitaria de la informática. Perspectivas, 2(1). 43-56.
}

Recibido: junio 03 de 2016

Aceptado: septiembre 20 de 2016

\begin{abstract}
Palabras clave
Recursos pedagógicos, espacios virtuales de aprendizaje, plataforma virtual Moodle, trabajo colaborativo
\end{abstract}

Resumen: El presente artículo describe las valoraciones que hacen los docentes sobre los usos de la plataforma virtual como un recurso de enseñanza y aprendizaje en los cursos de informática de la Universidad de Santander, sede Cúcuta. Para la recolección de la información necesaria para la investigación se empleó una encuesta aplicada a ocho profesores pertenecientes al Departamento de Sistemas de la Universidad. Esta encuesta se complementó con una entrevista semiestructurada centrada en las experiencias subjetivas de tres docentes. Entre los resultados más relevantes aparece que los profesores que participaron en el estudio utilizan frecuentemente la plataforma virtual como complemento para el desarrollo de sus cursos. El manejo apropiado de la plataforma se lleva a cabo como un proceso dinámico e interactivo de tal manera que el profesor constituye la base fundamental de la construcción de ideas en su entorno y muestra que el uso de la plataforma virtual en la formación de los estudiantes universitarios ha logrado que se reflexione sobre las técnicas elementales de la educación y puedan ser aplicadas en los procesos de enseñanza basados en la formación virtual.

*Autor para correspondencia

ju.claro@mail.udes.edu.co 


\section{Keywords}

Pedagogical resources, virtual learning spaces, Moodle virtual platform, collaborative work

\section{Palavras chave \\ Recursos pedagógicos, espaços virtuais de aprendizagem, plataforma virtual Moodle, trabalho colaborativo}

\section{Assessment of the use of the Moodle virtual platform as a pedagogical resource in the university teaching of computer science}

\begin{abstract}
This article describes teachers' assessments regarding the uses of the virtual platform as a teaching and learning resource in informatics courses of the University of Santander, Cúcuta. As a means of collecting the information necessary for the investigation, a survey of eight professors belonging to the Systems Department of the university was carried out. This survey was complemented by a semistructured interview centered on the subjective experiences of three teachers. Among the most relevant results, it appears that the professors who participated in the study frequently use the virtual platform as a complement for the development of their courses. The appropriate management of the platform is executed as a dynamic and interactive process, in such a way that the teacher functions as the fundamental basis for the construction of ideas in its environment. Furthermore, it demonstrates that the use of the virtual platform in the training of university students has managed to reflect on the elementary techniques of education and can be applied in teaching processes based on virtual training.
\end{abstract}

\section{Avaliação do uso da plataforma virtual Moodle como recurso pedagógico no ensino universitário de informática}

Resumo: Este artigo descreve as avaliações feitas pelos professores sobre os usos da plataforma virtual como recurso de ensino e aprendizagem nos cursos de informática da Universidade de Santander, Cúcuta. Para a recolha das informações necessárias para a investigação, foi utilizada uma pesquisa aplicada a oito professores pertencentes ao Departamento de Sistemas da Universidade. Esta pesquisa foi complementada por uma entrevista semi-estruturada centrada nas experiências subjetivas de três professores. Entre os resultados mais relevantes, parece que os professores que participaram no estudo freqüentemente usam a plataforma virtual como complemento para o desenvolvimento de seus cursos. O gerenciamento apropriado da plataforma é realizado como um processo dinâmico e interativo, de tal forma que o professor é a base fundamental da construção de idéias em seu ambiente e mostra que o uso da plataforma virtual na formação de estudantes universitários conseguiu refletir sobre as técnicas elementares de educação e pode ser aplicado em processos de ensino baseados em treinamento virtual. 


\section{Introducción}

El uso de Espacios Virtuales de Aprendizaje (EVA) gana cada día terreno en las instituciones educativas y en todos los niveles y modalidades, bien sea como complemento a las actividades presenciales o como una herramienta única a partir de la cual se desarrollan los contenidos de aprendizaje.

En la comunidad académica, el uso de las herramientas de Internet $y$ de otras redes de telecomunicaciones como recursos para la enseñanza es conocida como e-learning. De acuerdo con Area y Adell (2009), el concepto de e-learning (o de otros similares como tele formación, educación virtual, cursos on line, enseñanza flexible, educación web o docencia en línea) es una modalidad de enseñanzaaprendizaje que consiste en el diseño, puesta en práctica y evaluación de un curso o plan formativo desarrollado a través de redes de ordenadores y puede definirse como una educación o formación ofrecida a individuos que están geográficamente dispersos o separados o que interactúan en tiempos diferidos del docente empleando los recursos informáticos y de telecomunicaciones"(p.2).

De esta manera el concepto incluye todas las fases del proceso educativo, esto es: planificación, enseñanza, evaluación y retroalimentación. Por eso, su manejo provechoso supone un alto grado de especialización en el conocimiento y manejo de estas herramientas.

Son varias las ventajas atribuidas al uso de EVA en la enseñanza: extensión de los estudios y formación fuera del aula, acceso constante a múltiples materiales y recursos, incremento en la autonomía del estudiante, flexibilización de horarios y trabajo colaborativo (Area y Adell, 2009). Otras ventajas atribuidas al uso de EVA están relacionadas con la creación de comunidades virtuales para el aprendizaje digital basadas en el intercambio y cooperación a través de sistemas de comunicación mediados por tecnologías (Salinas, 2003). Sin embargo, también los docentes requieren cierto nivel de formación e inversión de tiempo y en consecuencia. Estos dos aspectos (obligación de formación e inversión de tiempo) pueden ser interpretados por algunos docentes como factores adversos a sus intereses personales y causar resistencia para adoptar el uso de los EVA.

Tal ha sido el impacto de las comunidades virtuales en la educación que ya hay quien habla de la necesidad de tipologías que permiten un mejor manejo conceptual de las mismas. Especialistas como Jonassen, Peck y Wilson (1999) y Salinas (2003) reconocen al menos cuatro categorías de estos espacios: de discurso, de práctica, de construcción del conocimiento y de aprendizaje. Estos mismos autores plantean un conjunto de aspectos mínimos que deben contemplar la estructura y organización de las comunidades de aprendizaje: la asignación de miembros, distribución de funciones y tareas de gestión.

Son variadas las plataformas virtuales de aprendizaje disponibles en el mercado, muchas con características y propósitos particulares y otras con funciones bajo estándares generales. Cesteros (2009) hace una relación importante acerca de la disposición de esta herramienta. Una característica importante en esta distinción está relacionada con el costo, en la que encontramos versiones libres y de pago.

Entre las plataformas libre de pago, una que goza de amplia aceptación en las comunidades académicas hispanas es la Moodle conocida, como una plataforma de aprendizaje a distancia (e-learning) basada en software libre. Igualmente, conocido es el sistema de gestión avanzada (también denominado Entorno Virtual de EnseñanzaAprendizaje (EVA), que no es otra cosa que una aplicación diseñada para ayudar a los educadores a crear cursos de calidad en línea. Estos tipos de sistema de aprendizaje a distancia a veces son también llamados Ambientes de Aprendizaje Virtual o Educación en Línea (CEP de Alcalá de Guadaíra,s/f)

Son numerosas las investigaciones que han reportado los beneficios y potencialidades del uso de Moodle como espacio virtual de aprendizaje, 
especialmente en el ámbito universitario (Casales, Rojas y Paulí. 2008; Marín y Madonado, 2011; Rivas, 2006; Machado y otros, 2012). Es así como el estudio de las estrategias, potencialidades y optimización del uso de esta plataforma es un tema de especial interés en las comunidades docentes de educación superior.

En relación con lo anterior, esta investigación ha sido destinada a la descripción de las valoraciones que hacen los docentes de los cursos de Informática sobre los usos de la plataforma virtual como un recurso de enseñanza y aprendizaje en los cursos de informática de la Universidad de Santander. Concretamente, se detallan las distintas valoraciones que hacen los profesores de esta herramienta como un recurso pedagógico para enseñar los contenidos de las asignaturas de esta área. Es importante tener en cuenta que desde el uso de esta plataforma de aprendizaje se logra formar a los estudiantes de los cursos de informática pertenecientes a la educación universitaria, lo que establece una postura importante para contribuir en el proceso de enseñanza que se encuentra presente en los ambientes virtuales de aprendizaje que se han venido afianzando en la universidad. Por lo tanto, la investigación encuentra pertinencia en cuanta contribución para la búsqueda de resultados positivos que permitan establecer el análisis de los fundamentos pedagógicos para la correcta utilización y manejo de esta plataforma educativa Moodle.

\section{Materiales y métodos}

La investigación que se propone presenta un enfoque cuantitativo que permitirá estudiar la relación entre variables cuantificadas. Según Pita Fernández y Pértegas Díaz (2002), “Los métodos cuantitativos son muy potentes en términos de validez externa ya que con una muestra representativa de la población hacen inferencia a dicha población a partir de una muestra con una seguridad y precisión definida". Esto significa que teniendo una cantidad de la población total se puede determinar la fuerza de asociación entre variables, logrando la objetividad de los resultados que pretenden buscar regularidades y la relación que explique el objeto de estudio. Con el fin de fortalecer los hallazgos cuantitativos se complementa el estudio con la aplicación de un análisis cualitativo, en el que se considera la opinión de algunos informantes clave sobre el uso de la plataforma Moodle como herramienta pedagógica. El nivel de la investigación es de carácter descriptivo, pues se busca caracterizar las variables asociadas el uso de espacios virtuales de aprendizaje entre los docentes entrevistados.

Para Hernández Sampieri, Fernández Collado y Baptista Lucio (2006), el enfoque cuantitativo consiste en la construcción y demostración de hipótesis que explican y predicen. Dicho con sus propias palabras, "la construcción con los estudios cuantitativos se pretende explicar y predecir los fenómenos investigados, buscando regularidades y relaciones causales entre elementos". Esto quiere decir que por medio de la encuesta se puede realizar la investigación, partiendo de una muestra representativa que exponga una parte fiable a menor escala de una población general. Por ello resulta esencial seleccionar bien la muestra de modo que esta garantice y represente la totalidad del colectivo a investigar.

Las hipótesis de investigación planteadas fueron las siguientes: a) Los docentes de los cursos de informática sienten temor de utilizar esta herramienta virtual en su labor docente porque tendrían que trabajar en dos escenarios, el aula de clase y el ambiente virtual, lo cual requeriría de un mayor tiempo de trabajo; b) Estos docentes consideran que a futuro esta herramienta los puede reemplazar en su labor como profesores de cátedra, con la eventual pérdida de su empleo.

\subsection{Participantes}

El escenario elegido ha sido la Universidad de Santander (UDES), ubicada en la ciudad de Cúcuta. La muestra seleccionada corresponde a 
una muestra exhaustiva, es decir, aquella que se considera apropiada para un estudio poblacional (censo). En razón de lo anterior, la encuesta fue aplicada a todos los individuos de una población, constituida en este caso por los ocho profesores pertenecientes al Departamento de Sistemas de la UDES Cúcuta, encargados de orientar presencial y virtualmente la asignatura de Informática. Para la selección de los docentes participantes en el estudio se tuvo en cuenta su vinculación como docentes de informática, su trabajo en la modalidad virtual y su manejo de la plataforma Moodle en la sede Cúcuta de la institución mencionada.

\subsection{Instrumento}

Se diseñó un instrumento útil para evaluar un conjunto indicadores relacionados con el uso de la plataforma Moodle: desde cuándo la usa, disponibilidad de recursos en el aula de clases, frecuencia de uso, satisfacción como usuario, importancia, eficiencia, impacto, rendimiento $\mathrm{y}$ responsabilidad del estudiante. Todo ítem se asoció a una escala tipo Likert que permitió medir en forma graduada cada indicador. El instrumento fue validado a través del juicio de expertos en el área y en los casos necesarios las preguntas se reformularon oportunamente. La fiabilidad del instrumento se aplicó a partir del modelo Alfa de Cronbach, que permitió determinar si cada ítem contribuye a medir eficazmente la variable que se pretende analizar.

La interpretación de los valores de confiabilidad se realizó tomando en cuenta la escala propuesta por Ruiz (1998): un valor comprendido dentro del rango 0.81-1.00, representa una confiabilidad muy alta. Al aplicar la prueba piloto se obtuvo un coeficiente alfa de Cronbach 0.98 . En consecuencia, los resultados obtenidos en la prueba presentan un rango de confiabilidad muy alto.

\subsection{Procedimiento}

Una vez aplicado el instrumento y presentados los resultados mediante la utilización del programa estadístico SPSS, se organizó la información con la respectiva tabulación, que fue registrada mediante gráficos de barras agrupadas. Con la finalidad de ampliar los resultados bajo el enfoque cuantitativo se complementó con un componente cualitativo. Para ello se utilizó la aplicación del software ATLASTI y como instrumento de recolección de información la entrevista semiestructurada, la cual se centró en las experiencias subjetivas de tres docentes con respecto al uso de la plataforma virtual Moodle. Durante su desarrollo se expusieron situaciones diversas de la cotidianidad de sus prácticas pedagógicas con el fin de dar claridad o ampliar la información sobre algunos aspectos relevantes mencionados en el componente cuantitativo.

\section{Resultados y discusión}

\subsection{Análisis de los resultados de la encuesta}

El conjunto de indicadores evaluados busca describir e interpretar la información relacionada con el uso de la plataforma virtual Moodle por parte de los docentes de informática de universidad UDES Cúcuta. La figura 1 muestra el tiempo que los docentes tienen usando la plataforma virtual en relación con su edad.

Figura 1
Tiempo de uso de la plataforma virtual Moodle en función de la edad de los docentes

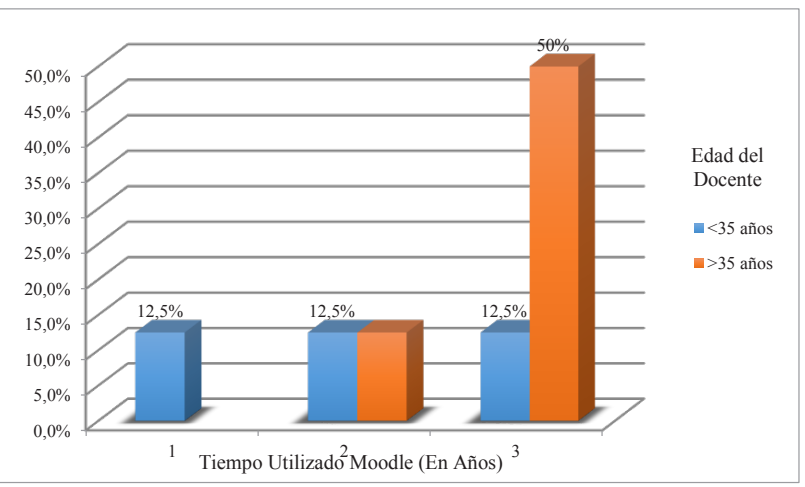

Fuente: Autor

Teniendo en cuenta la edad de los docentes y el tiempo que llevan utilizando la plataforma se puede observar lo siguiente: El $62.5 \%$ de los profesores tiene al menos dos años usando la herramienta; de 
este conjunto, $50 \%$ tiene más de 35 años y el $12.5 \%$ son menores. El 25\% de los encuestados tienen un año utilizando esta plataforma virtual: la mitad de los nuevos usuarios corresponde a profesores mayores a 35 años y la otra mitad son menores. También se refleja en la figura que hay un $12.5 \%$ que no utiliza Moodle como entorno virtual de aprendizaje y, que este grupo está conformado por profesores mayores de 35 años. En general se puede afirmar que la edad del docente no interfiere con el uso, pues el 87.5\% de los profesores está trabajando sus clases con la plataforma.

Figura 2

Disponibilidad de medios tecnológicos que permiten el desarrollo de las clases de informática utilizando la plataforma Moodle

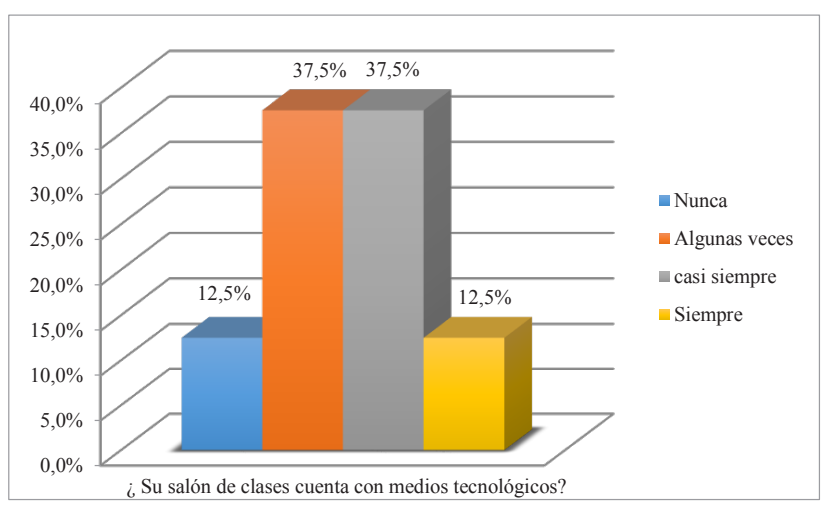

Fuente: Autor

La figura 2 muestra la adecuación de las aulas a los medios tecnológicos necesarios para el uso por los docentes de la plataforma Moodle. De acuerdo con las respuestas de los encuestados, el $87.5 \%$ de las aulas de clase cuentan siempre con los medios tecnológicos adecuados para desarrollar las clases de informática: el $12.5 \%$ restante afirma que disponen de ellos casi siempre. Del conjunto de las respuestas se colige que las salas de informática de la universidad cuentan con los medios adecuados para desarrollar las clases. Igualmente se evidencia que el $87.5 \%$ de los profesores desarrollan sus clases utilizando la plataforma y solo el $12.5 \%$ prescinde del recurso.
Figura 3

Frecuencia de uso de la plataforma Moodle para apoyar la labor docente e incorporación de actividades.

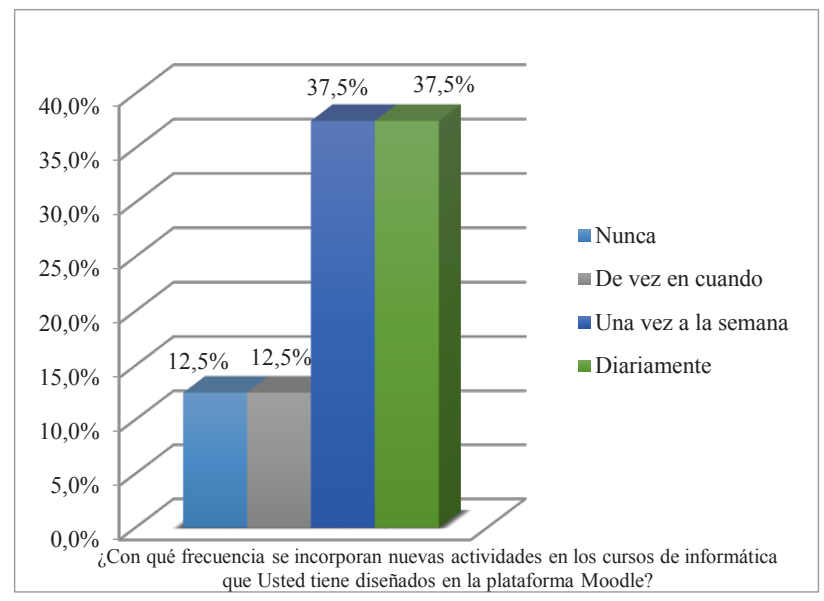

Fuente: Autor

En relación con la frecuencia de uso de la plataforma, los resultados presentados en la figura 3 evidencian que el $87,5 \%$ de los docentes usa Moodle para apoyar la labor en el aula, el 37.5\% la utiliza diariamente, otro $37.5 \%$ la emplea una vez a la semana y el $12.5 \%$ de vez en cuando. La gran mayoría de los docentes utiliza, pues, esta herramienta como complemento para su labor como profesor; sólo el $12.5 \%$ expresó que nunca la han utilizado en su actividad docente.

Figura 4

Satisfacción con el uso plataforma Moodle en las clases.

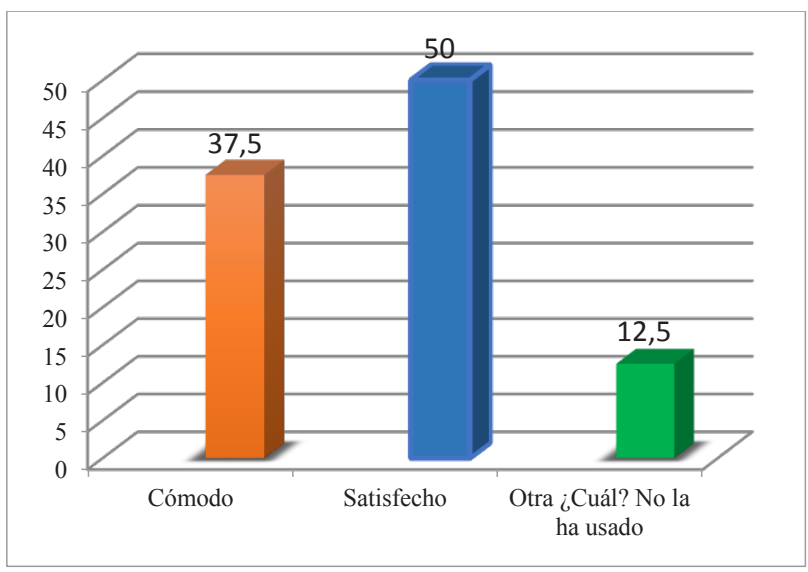

Fuente: Autor 
La figura 4 refleja la satisfacción de los docentes con el uso de la plataforma. Los resultados evidencian que el $87,5 \%$ de los docentes de informática se sienten en gran medida satisfechos cuando la usan en sus clases; específicamente, el 50\% de los profesores consideran sentirse satisfechos y el $37.5 \%$ manifiesta estar cómodo con el uso de esta herramienta en su labor como docente. El grado de confort del docente con el uso de la plataforma, por lo tanto, es el adecuado. Igualmente se muestra en el análisis que el $12.5 \%$ de profesores no se sienten agradados simplemente porque no la han usado.

Figura 5

Importancia de la plataforma Moodle como herramienta de aprendizaje

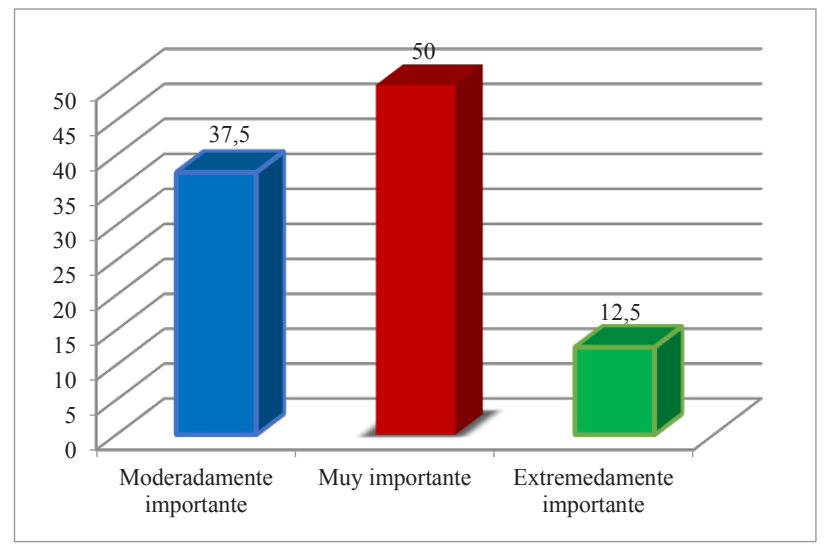

Fuente: Autor

En referencia a la valoración de la importancia que otorgan los docentes de informática a la plataforma Moodle como herramienta de aprendizaje, en la figura 5 se visualiza que para el $62,5 \%$ la plataforma es un aula virtual de aprendizaje al que atribuyen un alto grado de importancia. El $37,5 \%$ restante manifiesta que su importancia es moderada. Esta valoración deja claro que los docentes consideran que este entorno virtual de aprendizaje constituye una herramienta fundamental para la educación de los estudiantes de un curso y contribuye al cumplimiento de las expectativas necesarias para enseñar.

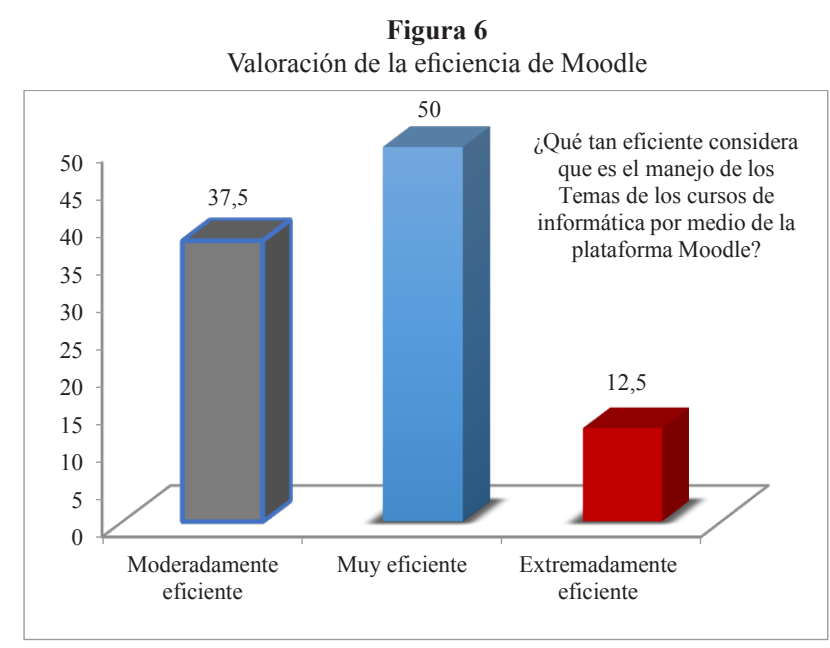

Fuente: Autor

La valoración de la eficiencia de la plataforma Moodle para cumplir los objetivos y la percepción de cuán eficiente es el manejo de los temas por medio de este entorno virtual se muestran en la figura 6 . De acuerdo con los resultados, el $62.5 \%$ de los docentes opina que la plataforma es importante para el cumplimiento de los objetivos propuestos de un curso y el $37.5 \%$ expresa que es moderadamente importante. Así pues, la plataforma es significativa para todos los docentes por cuanto contribuye con el cumplimiento de los objetivos trazados al iniciar las clases. Teniendo en cuenta los porcentajes del gráfico se desprende que la plataforma es para los docentes un entorno adecuado en cuyos espacios, recursos y actividades contribuyen al logro de los objetivos establecidos por el programa de un curso.

Figura 7

Valoración del impacto del uso de la plataforma

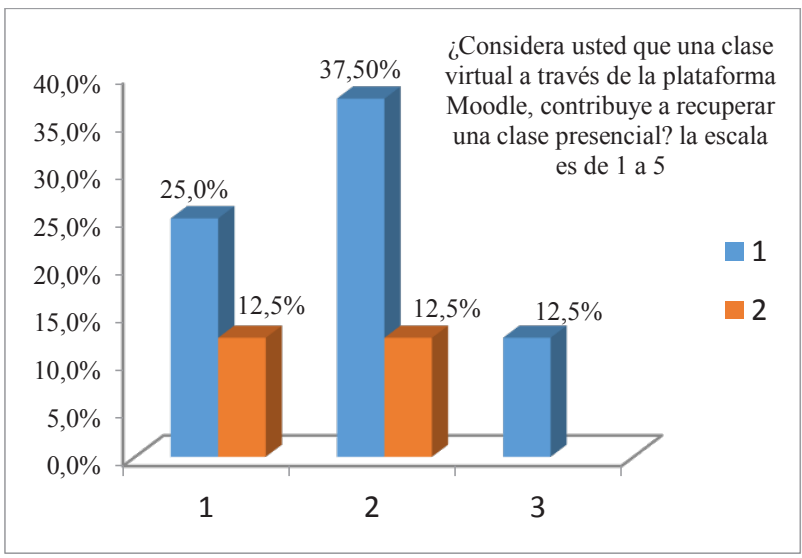

Fuente: Autor 
En la figura 7 se muestran los resultados sobre la percepción de los docentes en cuanto al impacto que ha tenido la plataforma en las actividades que contribuyen a la comprensión de los contenidos de los cursos y su apoyo a la clase presencial. En la plataforma se presentan varios tipos de actividades que pueden contribuir a la comprensión de los contenidos del curso. Sobre este particular, el 25\% de los profesores expresan que es mucho lo que aportan estas actividades y el 50\% consideran que son apropiadas. Por otra parte, un $25 \%$ de docentes estiman que la herramienta cumple con las expectativas para que los puedan entender las clases trabajadas en el entorno virtual. En suma, se puede afirmar que todos los docentes están de acuerdo con que las actividades de la plataforma son útiles para comprender las temáticas tratadas en un curso. A la pregunta de si una clase presencial puede ser recuperada por una clase virtual a través de la plataforma, el $12.5 \%$ de los docentes expresa que es mucho lo que contribuye a recuperar una clase que no se haya podido desarrollar en el aula de clase, un 50\% manifiestan que la plataforma cuenta con las condiciones apropiadas y el $37.5 \%$ restante de los profesores creen que está cumpliendo las expectativas que se necesitan para desarrollar una clase en su entorno. Esto quiere decir que el 100\% de los encuestados manifiestan estar conformes con la plataforma en el momento de utilizarla para desarrollar una clase virtual.

Figura 8

Valoración de la contribución del uso de la plataforma Moodle a la mejora del rendimiento estudiantil y al trabajo colaborativo

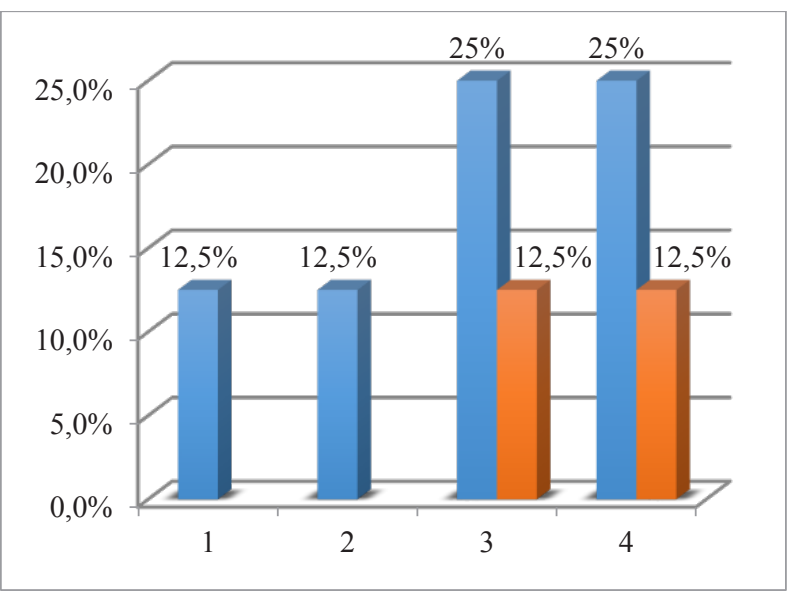

Fuente: Autor
La figura 8 relaciona las opiniones que hacen referencia a la contribución que se reconoce a la plataformaparamejorarelrendimientodelosalumnos y la valoración del grado en que se potencia el hecho de que los estudiantes participen constantemente orientados hacia al trabajo cooperativo. Con respecto a este particular, se evidencia que el 12,5\% de los profesores expresa que el rendimiento de los estudiantes mejora significativamente cuando se desarrolla un curso utilizando la plataforma, el $37.5 \%$ manifiestan que sí se percibe la mejora en el desempeño de los alumnos, el $25 \%$ creen que la plataforma alcanza a cumplir con este propósito y el $25 \%$ restante opinan que no es mucho lo que se mejora utilizando Moodle. En otras palabras, el $75 \%$ de los docentes encuestados están de acuerdo con que el uso de la plataforma virtual mejora el rendimiento de los estudiantes, en tanto que el $25 \%$ no se siente muy convencido de que la educación de los alumnos es mejor cuando se utiliza el aula virtual de aprendizaje. Teniendo en cuenta el hecho de que los estudiantes de los cursos participen activamente en la plataforma los conduce al trabajo cooperativo, se puede observar que el $37.5 \%$ de los profesores manifiestan que por medio de la plataforma sí se orienta a los alumnos a realizar sus actividades en grupo, otro $37.5 \%$ creen que la plataforma cumple con este propósito y un $12.5 \%$ manifiestan no estar muy convencidos; por otra parte, el $12.5 \%$ expresa que esta situación rara vez ocurre. Resumiendo, el $75 \%$ de los profesores sienten que la participación constante de los alumnos en el aula virtual de aprendizaje contribuye al trabajo cooperativo.

Finalmente, la figura 9 contiene los datos sobre la evaluación de los docentes sobre si la plataforma permite la comunicación virtual con los integrantes de los cursos, es decir, si representa un motivo que contribuye a la responsabilidad y autonomía de cada estudiante. El 25\% de los profesores considera que sí se aporta significativamente al fortalecimiento de estas cualidades, el $37.5 \%$ expresan que frecuentemente ayuda y otro $37.5 \%$ creen que su contribución es escasa. Dicho de otro modo, el $62.5 \%$ de los docentes manifiesta que el aula virtual 
de aprendizaje ayuda a que los estudiantes sean responsables y autónomos con sus tareas a través de la comunicación online que ofrece; el $37.5 \%$ restante opina que es muy poco lo que aporta esta forma de tener contacto con los integrantes de los cursos para generar compromiso individual.

Figura 9

Aportes de la plataforma a la responsabilidad y autonomía del estudiante

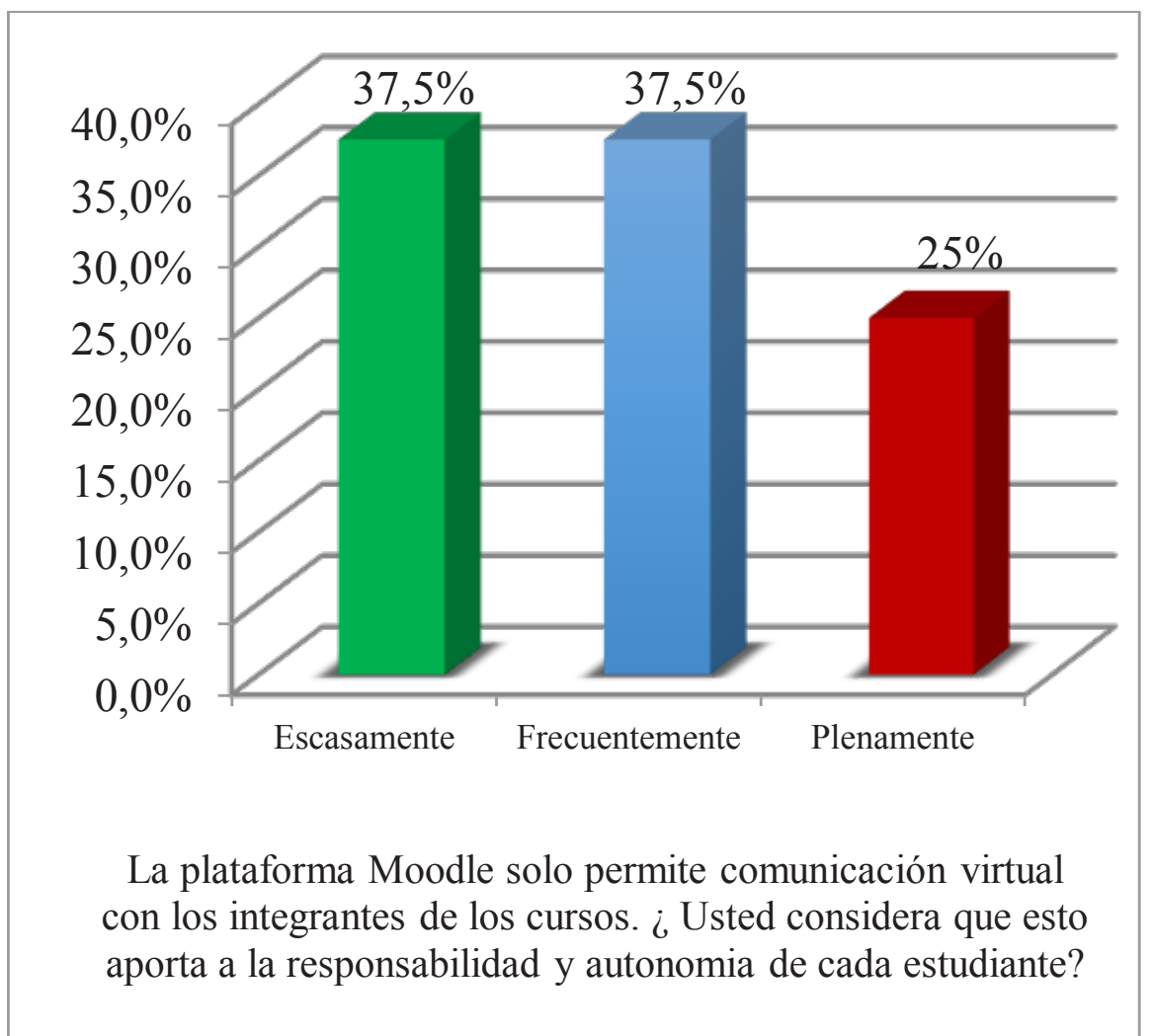

Fuente: Autor

\subsection{Análisis de los resultados de la entrevista}

El análisis de estas categorías se efectuó por medio de la entrevista, de las que se rescatan las concepciones que tiene cada profesor sobre el uso de la plataforma virtual. A partir de la codificación teórica aplicada con el programa Atlas/Ti se logró generar una estructuración de todas las categorías encontradas en las entrevistas realizadas a los tres docentes del área de informática de la Universidad de Santander que se evidencian en la Tabla 1.
Las categorías hacen referencia a la importancia pedagógica del uso de la herramienta como un recurso de aprendizaje que apoya el cumplimiento de los objetivos y fortalecimiento de las estrategias deseadas. Por otra parte, se destaca el perfil de los usuarios y de la interfaz y de las ventajas que de despliegan a partir del uso de la herramienta. 
Tabla 1

Categorías emergentes y significados principales generados a partir de la entrevista
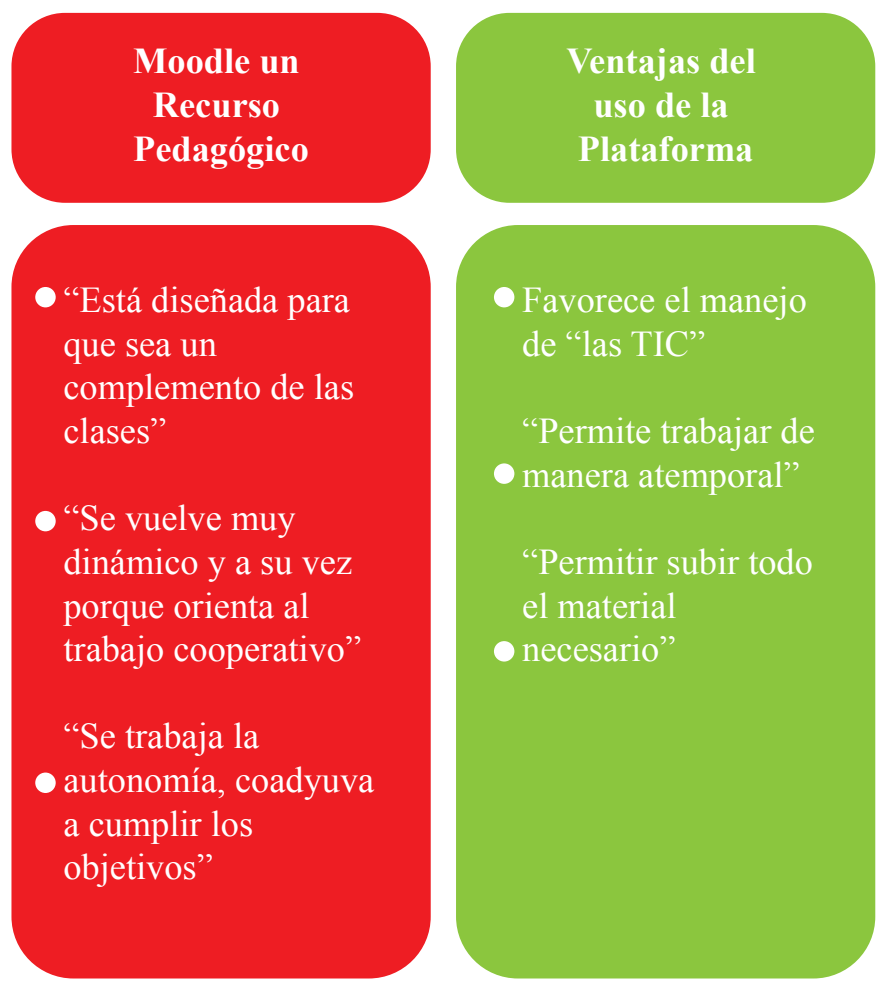
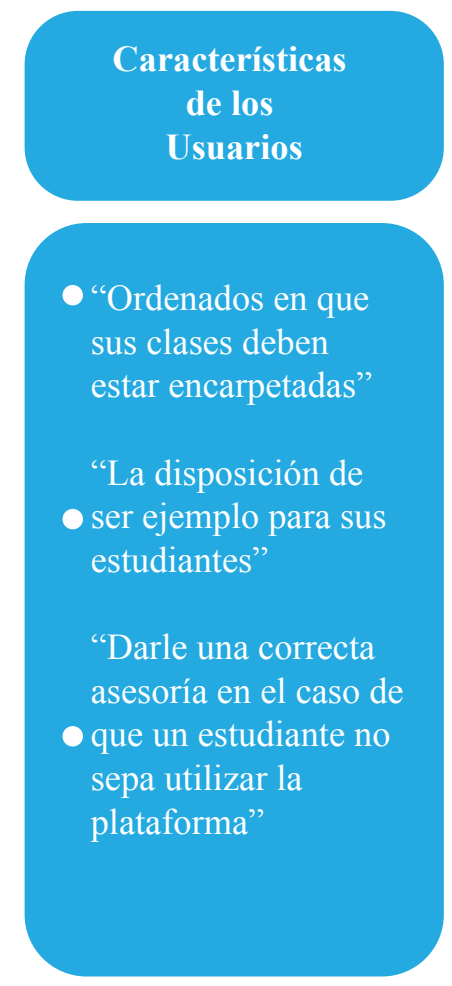
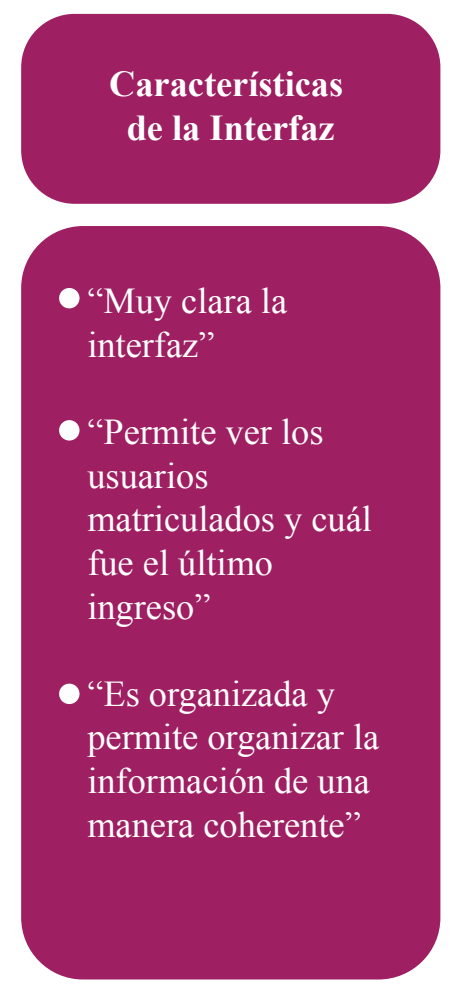

Fuente: Autor

\section{Discusión}

Los resultados de la investigación muestran que un número importante de docentes de los que imparten la informática como asignatura dentro de la UDES, Cúcuta, utiliza la plataforma Moodle como complemento para el desarrollo de sus clases. La edad parece no constituir ni obstáculo ni aliciente para utilizar la herramienta. Los docentes señalan que la plataforma no es de difícil manejo; por el contrario, resulta cómodo impartir conocimiento con las facilidades que ella proporciona. Esto coincide con lo que plantea Correa (2005) cuando afirma que el entorno del aula virtual de aprendizaje está diseñado para permitir el uso apropiado de esta herramienta.

Se evidencia, por otra parte, que la universidad UDES, sede Cúcuta, cuenta con los medios apropiados para apoyar las clases usando la plataforma Moodle. Ya que existen las condiciones materiales y tecnológicas, y vista su utilidad e importancia, habría que motivar al personal docente que no se vale del aula virtual para que incorpore a sus estrategias pedagógicas esta poderosa herramienta. En este sentido, los profesores que la emplean tienen conciencia de la plataforma Moodle está diseñada para complementar las clases y que, por tanto, representa un aporte para contribuir al mejor desempeño del trabajo realizado por el docente en el aula de clase. Dicho de otro modo, el aula virtual no sustituye al profesor ni anula la relevancia de sus clases presenciales, sino que supone un complemento. En este sentido se pronuncia Velásquez (2012) cuando señala que es fundamental comprometerse en el buen uso de las herramientas virtuales en cuanto aportan mucho a 
la educación superior, en las que es preciso innovar conforme avanzan las nuevas tecnologías a fin de contribuir con la formación de los estudiantes.

Los profesores de informática manifiestan en un alto porcentaje que usan la plataforma Moodle para apoyar la labor docente, lo cual indica que un buen grupo de profesores está cumpliendo con el uso de la plataforma para el desarrollo de los cursos de informática. La frecuencia con que se incorporan nuevas actividades, documentos o archivos que ayudan al proceso de enseñanza y aprendizaje de los estudiantes de los cursos de informática está relacionada con la importancia que el docente le da al uso de la plataforma Moodle. Haciendo referencia a las facilidades y apoyos que proporcionan a los profesores en su labor, Segura (2009) señala que la creación de actividades y la adición de material de apoyo a través del aula virtual de aprendizaje es una de tantas funciones que esta permite. La importancia que el docente otorga a estas tareas debe ser de acuerdo a las necesidades formativas que se tengan para mejorar el aprendizaje del estudiante.

De esta forma, los docentes señalan que la plataforma Moodle presenta entre sus opciones la posibilidad de crear un foro, un chat o una encuesta, todo lo cual ayuda a valorar el rendimiento de un estudiante y supone un aporte significativo para los docentes por cuanto las actividades propuestas están dispuestas para ser utilizadas en el momentos en que ellos las requieran. En este sentido, y de acuerdo con el juicio de los profesores, estas actividades se proponen para fortalecer las competencias de los estudiantes.

Para la mayoría de los docentes del área de informática de la UDES es muy importante trabajar con la plataforma Moodle como herramienta de aprendizaje. De hecho, están satisfechos con los recursos que la plataforma les ofrece para el manejo de los temas y contenidos que deben desarrollar durante las clases. De igual manera se aprecia que los docentes expresan que la plataforma Moodle contribuye con la educación presencial y, de acuerdo a su creación y diseño, funciona como un aula virtual de aprendizaje que beneficia la educación de los estudiantes. En relación con lo anterior Correa (2005), hace énfasis en que la plataforma ayuda a la organización de los contenidos, siendo este un aporte muy importante de la herramienta para contribuir con la enseñanza que se da en la universidad, pues brinda estrategias alternativas de enseñanza-aprendizaje fundamentadas en las nuevas tecnologías de la informática y de la comunicación.

La plataforma, en efecto, permite que el docente se valga de diferentes técnicas y métodos de enseñanza para que el estudiante pueda demostrar sus capacidades. Una muestra de las posibilidades pedagógicas de la plataforma se encuentra en el caso de los talleres que se diseñan durante el semestre; a través de Moodle y de manera muy fácil se ofrecen al alumno todos los contenidos que se quieran exponer a través de todos los recursos que la herramienta ofrece. Baños (2007) es de esta opinión, pues señala que la plataforma Moodle facilita el acceso a los recursos del aula virtual para que se pueda organizar todo el material de apoyo en su entorno, lo cual contribuye a que el docente se sienta cómodo al momento de programar sus temas.

Para un alto porcentaje de los docentes, una clase virtual a través de la plataforma Moodle permite recuperar una clase presencial. Sin embargo, señalan que es importante tener claridad con respecto a las reglas de juego que se acuerden entre el profesor y el estudiante, y también consideran que el profesor debe ser ordenado con el tema y los recursos que necesita el aprendiz. En relación con lo anterior, Carneiro, Toscano y Díaz (2009) señalan que las tecnologías de la informática y de la comunicación han contribuido a que el aprendizaje se pueda dar en cualquier parte y en cualquier momento sin necesidad de estar en el aula de clase y estas herramientas han sido diseñadas para brindar las garantías necesarias de la educación del sujeto que las utiliza.

Por otra parte, los docentes de área de informática que trabajan con la plataforma Moodle para apoyar su labor docente consideran que cuando los estudiantes de los cursos participan activamente en ella fortalecen la capacidad del 
trabajo cooperativo. Teniendo en cuenta lo descrito inicialmente, se evidencia que los profesores opinan que Moodle como recurso pedagógico es fundamental, porque fomenta el trabajo cooperativo, dinámico y esto se demuestra a través de los foros cuando los estudiantes aportan ideas. En este orden de ideas, Velásquez (2012) afirma que los entornos virtuales tienen la función de favorecer el aprendizaje responsable y cooperativo de los participantes. Pero se debe tener en cuenta que los profesores son los encargados de dirigir el manejo del aula virtual a fin de que los estudiantes aprendan a ser responsables en el cumplimiento de sus actividades.

Para la gran mayoría de los docentes participantes en el estudio, la plataforma Moodle no solo permite la comunicación virtual con los integrantes de los cursos, sino que además les ayuda a fortalecer la responsabilidad y a la autonomía de cada uno de ellos. En relación con lo anterior, dicen los profesores de informática que es fundamental la responsabilidad que Moodle permite desarrollar en los estudiantes ya que ellos se muestran más autónomos al momento de aprender porque tienen la posibilidad de trabajar de manera libre y pueden realizar los talleres desde su casa o en el aula de clase si es el caso. Sobre este particular se pronuncia Velásquez (2012), quien comenta que el aula virtual de aprendizaje fomenta el trabajo individual de los integrantes y de igual manera su entorno permite que a través de la comunicación online se puedan desarrollar y planificar modelos de enseñanza más flexibles y accesibles que contribuyan con la formación de alumnos.

\section{Conclusiones}

Los docentes de los cursos de informática de la Universidad de Santander asumen el uso de la plataforma virtual Moodle bajo el convencimiento de que esta herramienta influye efectivamente en el trabajo del docente para mejorar las habilidades de los estudiantes en sus diferentes ambientes de aprendizaje.
La plataforma Moodle busca transformar los procesos de enseñanza y aprendizaje en prácticas que promuevan el uso apropiado de las TIC como herramientas de apoyo para la educación. De esta manera los docentes pueden mostrar las habilidades necesarias a sus estudiantes para poder solucionar dificultades, trabajar en forma colaborativa, tener acceso a la información, logrando participar en un entorno seguro que esté fundamentado en el uso responsable de la tecnología.

La plataforma Moodle permite innovar las estrategias pedagógicas y el trabajo de aula en el sentido de que por su intermedio se promueve el desarrollo y fortalecimiento de habilidades digitales entre los docentes y de igual manera; de igual manera, se propicia el uso apropiado de las TIC en beneficio de un aprendizaje constructivo y significativo, que favorezca a los estudiantes en la adquisición de competencias en el uso de las tecnologías.

El aula virtual de aprendiza Moodle enriquece la práctica pedagógica en el aula, pues contribuye con el desarrollo de habilidades tecnológicas en los estudiantes a partir del uso de las TIC como herramientas apropiadas en las actividades de enseñanza. Igualmente permite conocer y aplicar diferentes técnicas que contribuyan a la investigación a partir de la información a la que se puede acceder por su intermedio.

El uso de la plataforma Moodle también resulta útil para la acción reflexiva sobre la aplicación educativa que ofrecen los diferentes ambientes virtuales de aprendizaje. De esta manera se promueve el desarrollo de habilidades en la utilización del programa, lo que contribuirá a mostrar buenas prácticas mediante el uso educativo y reflexivo. Asimismo apoya al profesor, pues permite el diseño de actividades para lograr que los estudiantes entiendan los temas y la información propia de los cursos de informática.

Se evidencia que hay una frecuencia de uso de la plataforma Moodle por parte de los docentes de informática y esto ha contribuido a que los estudiantes expresen sus habilidades de manejo de Moodle para el desempeño y rendimiento 
personal. Con esto se logra una participación de trabajo cómodo que permite visualizar y realizar las actividades propuestas en la plataforma virtual.

Los docentes que utilizan la plataforma Moodle se integran en una propuesta educativa basada en el aprendizaje b-learning, que tiene como finalidad enriquecer las clases presenciales de manera que se facilite la comunicación e información entre el profesor y los estudiantes de los cursos de informática.

La evaluación global sobre Moodle es bastante positiva para los docentes de informática de la UDES, pues es considerada como una herramienta que permite trabajar desde un enfoque constructivista en los contextos universitarios. El manejo apropiado de la plataforma se lleva a cabo como un proceso dinámico e interactivo de tal manera que el profesor se constituye en base fundamental de la construcción de ideas que apoyan el uso del aula virtual de aprendizaje.

\section{Referencias}

Area, M., y Adell, J. (2009). E-learning: enseñar y aprender en espacios virtuales. En De Pablos, J. (Coord.).Tecnología educativa. La formación del profesorado en la era de Internet. Málaga: Aljibe, págs. 391-424

Baños, J. (2007), La Plataforma Educativa Moodle, Creación de Aulas Virtuales. Manual de consulta para el profesorado (Versión 1.8). Disponible en http://tice.wikispaces.com/file/view/Moodle18 Manual_Prof-p1.pdf

Carneiro, R.; Toscano, J.C. y Díaz, T. (2009). Los desafíos de las TIC para el cambio educativo. Madrid: OEI - Fundación Santillana. Disponible en http://www.oei.es/metas2021/LASTIC2.pdf

Casales, R., Rojas, J., y Paulí, G. (2008). Algunas experiencias didácticas en el entorno de la plataforma Moodle. Revista de informática educativa y medios audiovisuales, 5(19): 1-10.

CEP de Alcalá de Guadaíra (Sevilla) (On line). Disponible en http://www.redes- epalcala.org/ plataforma/file.php/1/manual_plataforma_cep_ alcala.pdf

Cesteros, A. F. P. (2009). Las plataformas e-learning para la enseñanza y el aprendizaje universitario en Internet. EnLópez Alonso, C. y Matesanz del Barrio, M.(Eds.). Las plataformas de aprendizaje: del mito a la realidad. Madrid:BibliotecaNueva.

Correa, J.M. (2005). La integración de plataformas de e-learning en la docencia universitaria: Enseñanza, aprendizaje e investigación con Moodle en la formación inicial del profesorado. Revista Latinoamericana de Tecnología Educativa, 3 (1): 37-48.

Hernández Sampieri, R., Fernández Collado, C. y Baptista Lucio, M. 2006. Metodología de la investigación. México: Mc Graw Hill.

Jonassen, D. H., Peck, K. y Wilson, B. G. (1999). Learning with technology: A constructivist approach. Upper Saddle River, NJ: Merrill.

Machado, A. M., López, R. B., Fanjul, N. J., Y Povedano, N. A. (2012). El foro en la plataforma Moodle: un recurso de la participación cooperativa para el aprendizaje de las matemáticas. EDMETIC, 1(2): 29-43.

Marín Díaz, V., y Maldonado Berea, G. A. (2011). El alumno universitario cordobés y la plataforma virtual Moodle. Pixel-Bit. Revista de Medios y Educación, (38): 121-128 .

Pita Fernández, S. y Pértegas Díaz, S. (2002). Investigación cuantitativa y cualitativa. Atención Primaria, 9: 76-78. Disponible en http://www. fisterra.com/mbe/investiga/cuanti_cuali/cuanti_ cuali2.pdf

Rivas, F. C. (2006). La plataforma de aprendizaje Moodle como instrumento para el trabajo social en el contexto del espacio europeo de la educación superior. Acciones e investigaciones sociales, (1 Ext), 367.

Ruiz, C. (1998), Instrumentos de Investigación Educativa. Procedimiento para su diseño yvalidación. Barquisimeto: CIDEC .

Salinas, J. (2003). Comunidades virtuales y aprendizaje digital. Edutec, 54(2): 1-21.

Segura, M. (2009). Plataformas Educativas y Redes 
Docentes. En Carneiro, R.; Toscano, J. y Díaz, T. (Eds.), Los desafíos de las TIC para el cambio educativo. Madrid: Fundación Santillana. pp. 95-109. Disponible en http://revistas.uptc.edu. co/index.php/educacion_y_ciencia/article/ view/3240/2917

Velásquez, A. (2012), Una mirada crítica a la didáctica de las ciencias, la educación científica y el papel de las Tic en la Educación Superior. Ibagué: Universidad De Tolima. 